PROCEEDINGS OF THE

AMERICAN MATHEMATICAL SOCIETY

Volume 134, Number 9, September 2006, Pages 2533-2540

S 0002-9939(06)08286-4

Article electronically published on March 23, 2006

\title{
ON $p$-ADIC HERMITIAN EISENSTEIN SERIES
}

\author{
SHOYU NAGAOKA \\ (Communicated by Wen-Ching Winnie Li) \\ Dedicated to Professor Yasuo Morita
}

\begin{abstract}
In this paper we generalize the notion of $p$-adic modular form to the Hermitian modular case and prove a formula that shows a coincidence between certain $p$-adic Hermitian Eisenstein series and the genus theta series associated with Hermitian matrix with determinant $p$.
\end{abstract}

\section{INTRODUCTION}

In a previous study [8], the present author generalized the notion of $p$-adic Eisenstein series to the Siegel modular case and found a "curious formula" $(8,(8.6))$ that shows a coincidence between $p$-adic Siegel-Eisenstein series and theta series. Strictly speaking, certain $p$-adic Siegel-Eisenstein series were shown to coincide with the genus theta series associated with a binary quadratic form of discriminant $-p$.

In another study [5], a more general type of $p$-adic Siegel-Eisenstein series was investigated. The results of both of these studies indicate that these kinds of $p$-adic Siegel-Eisenstein series become "true" modular forms, namely, ordinary modular forms of $\Gamma_{0}(p)$-type.

In this note, we shall extend these findings in a different direction. Namely, we show that a similar phenomenon also occurs in the Hermitian modular case.

\section{DEFINITION AND NOTATION}

2.1. Hermitian modular forms. Let $R$ be a subring of $\mathbb{C}$. We shall denote by $\operatorname{Her}_{m}(R)$ the space of $m$ by $m$ Hermitian matrices over $R$ with respect to the complex conjugate. Let $\operatorname{Her}_{m}^{+}(R)$ be the subset of $\operatorname{Her}_{m}(R)$ consisting of positive elements. The space

$$
\mathbb{H}_{n}:=\left\{Z \in M_{n}(\mathbb{C}) \mid \frac{1}{\sqrt{-1}}\left(Z-{ }^{t} \bar{Z}\right) \in \operatorname{Her}_{n}^{+}(\mathbb{C})\right\}
$$

is called the Hermitian upper-half space of degree $n$, where ${ }^{t} \bar{Z}$ denotes the complex conjugate, transpose matrix of $Z$. The group

$$
G_{n}=U(n, n):=\left\{M \in M_{2 n}(\mathbb{C}) \mid{ }^{t} \bar{M} J_{n} M=J_{n}, J_{n}=\left(\begin{array}{rr}
0 & 1_{n} \\
-1_{n} & 0
\end{array}\right)\right\}
$$

acts on $\mathbb{H}_{n}$ by the ordinary generalized linear fractional transformation.

Received by the editors December 17, 2004 and, in revised form, April 7, 2005.

2000 Mathematics Subject Classification. Primary 11F33; Secondary 11F55.

Key words and phrases. p-adic modular forms, Eisenstein series.

(C)2006 American Mathematical Society Reverts to public domain 28 years from publication 
Let $\mathbb{K}$ be an imaginary quadratic field of discriminant $d_{\mathbb{K}}$ with the ring of integers $\mathcal{O}_{\mathbb{K}}$. The Hermitian modular group over $\mathbb{K}$ is defined by $\Gamma_{n}(\mathbb{K}):=G_{n} \cap M_{2 n}\left(\mathcal{O}_{\mathbb{K}}\right)$. We denote by $M_{k}\left(\Gamma_{n}(\mathbb{K})\right)$ the complex vector space consisting of Hermitian modular forms of weight $k$ for $\Gamma_{n}(\mathbb{K})$.

Let $f(Z)$ be a Hermitian modular form in $M_{k}\left(\Gamma_{n}(\mathbb{K})\right)$. Then, $f(Z)$ has a Fourier expansion of the form

$$
f(Z)=\sum_{0 \leq H \in \Lambda_{n}(\mathbb{K})} a_{f}(H) \exp [2 \pi \sqrt{-1} \operatorname{tr}(H Z)], \quad Z \in \mathbb{H}_{n},
$$

where the index set $\Lambda_{n}(\mathbb{K})$ is defined by

$$
\Lambda_{n}(\mathbb{K}):=\left\{H=\left(h_{i j}\right) \in \operatorname{Her}_{n}(\mathbb{K}) \mid h_{i i} \in \mathbb{Z}, \sqrt{d_{\mathbb{K}}} h_{i j} \in \mathcal{O}_{\mathbb{K}}(i \neq j)\right\} .
$$

\subsection{Hermitian Eisenstein series. Define}

$$
\Gamma_{n}(\mathbb{K})_{\infty}:=\left\{\left(\begin{array}{ll}
A & B \\
C & D
\end{array}\right) \in \Gamma_{n}(\mathbb{K}) \mid C=O_{n}\right\} .
$$

For an integer $k$ such that $k>2 n$ and $w_{\mathbb{K}} \mid k\left(w_{\mathbb{K}}\right.$ is the order of the unit group of $\mathbb{K})$, define a series given by

$$
G_{k}^{(n)}(Z):=\sum_{\substack{* * \\ C}) \in \Gamma_{n}(\mathbb{K})_{\infty} \backslash \Gamma_{n}(\mathbb{K})} \operatorname{det}(C Z+D)^{-k}, \quad Z \in \mathbb{H}_{n} .
$$

This series belongs to $M_{k}\left(\Gamma_{n}(\mathbb{K})\right)$ and is called the Hermitian Eisenstein series of weight $k$ for $\Gamma_{n}(\mathbb{K})$. It was shown by Braun [3] that all of the Fourier coefficients of $G_{k}^{(n)}$ are rational.

2.3. Genus theta series. Let $S \in \operatorname{Her}_{m}^{+}\left(\mathcal{O}_{\mathbb{K}}\right)$. Define

$$
\Theta^{(n)}(S ; Z):=\sum_{X \in M_{m, n}\left(\mathcal{O}_{\mathbb{K}}\right)} \exp [\pi \sqrt{-1} \operatorname{tr}(S[X] Z)], \quad Z \in \mathbb{H}_{n},
$$

where $S[X]:={ }^{t} \bar{X} S X$. Let $\left\{S_{1}, \ldots, S_{h}\right\}$ be a set of representatives of unimodular equivalence classes of the genus containing $S$. The genus theta series associated with $S$ is defined by

$$
\text { genus } \Theta^{(n)}(S)(Z):=\left(\sum_{i=1}^{h} \frac{\Theta^{(n)}\left(S_{i} ; Z\right)}{E\left(S_{i}\right)}\right) /\left(\sum_{i=1}^{h} \frac{1}{E\left(S_{i}\right)}\right),
$$

where $E\left(S_{i}\right)$ is the order of the unit group of $S_{i}$.

2.4. $p$-adic Hermitian Eisenstein series. Put $\omega=\left(d_{\mathbb{K}}+\sqrt{d_{\mathbb{K}}}\right) / 2$ and define the matrices $\dot{Z}=\left(\dot{z}_{i j}\right)$ and $\ddot{Z}=\left(\ddot{z}_{i j}\right)$ by

$$
\dot{Z}:=\frac{\omega^{t} Z-\bar{\omega} Z}{\omega-\bar{\omega}}, \quad \ddot{Z}:=\frac{Z-{ }^{t} Z}{\omega-\bar{\omega}} .
$$

Thus, any Hermitian modular form $f(Z)$ can be considered as a function of $n(n-1) / 2$ complex variables $\ddot{z}_{i j}(i<j)$ in $\ddot{Z}$ and $n(n+1) / 2$ complex variables $\dot{z}(i \leq j)$ in $\dot{Z}$. If we put

$$
\dot{q}_{i j}:=\exp \left(2 \pi \sqrt{-1} \dot{z}_{i j}\right)(i \leq j), \quad \ddot{q}_{i j}:=\exp \left(2 \pi \sqrt{-1} \ddot{z}_{i j}\right)(i<j),
$$

then

$$
q^{H}:=\exp [2 \pi \sqrt{-1} \operatorname{tr}(H Z)]=\prod_{i \leq j} \dot{q}_{i j}^{\xi_{i j}} \prod_{i<j} \ddot{q}_{i j}^{\eta_{i j}},
$$


where $H=\left(h_{i j}\right)$ and

$$
\xi_{i i}=h_{i i}, \xi_{i j}=h_{i j}+\bar{h}_{i j}(i<j), \eta_{i j}=\bar{\omega} h_{i j}+\omega \bar{h}_{i j}(i<j)
$$

are rational integers. Based on the semi-positivity of $H$, we may regard a Hermitian modular form $f(Z)$ as an element of a formal power series ring

$$
\mathbb{C}\left[\dot{q}_{i j}^{ \pm}, \ddot{q}_{i j}^{ \pm}\right]\left[\left[\dot{q}_{11}, \ldots, \dot{q}_{n n}\right]\right] .
$$

Let $\left\{k_{m}\right\}_{m=1}^{\infty}$ be a sequence of increasing natural numbers. If the corresponding sequence of Hermitian Eisenstein series $\left\{G_{k_{m}}^{(n)}\right\} \subset \mathbb{Q}\left[\dot{q}_{i j}^{ \pm}, \ddot{q}_{i j}^{ \pm}\right]\left[\left[\dot{q}_{11}, \ldots, \dot{q}_{n n}\right]\right]$ converges $p$-adically to an element of $\mathbb{Q}_{p}\left[\dot{q}_{i j}^{ \pm}, \ddot{q}_{i j}^{ \pm}\right]\left[\left[\dot{q}_{11}, \ldots, \dot{q}_{n n}\right]\right]$, we shall call the limit $\lim _{m \rightarrow \infty} G_{k_{m}}^{(n)}$ a $p$-adic Hermitian Eisenstein series ([10] and [8], (5.3)).

\section{MAin Result}

In the remainder of this note, we will restrict ourselves to the case in which

$$
\mathbb{K}=\mathbb{Q}(\sqrt{-1}) \text { : the Gaussian field. }
$$

In this case, we have $\mathcal{O}_{\mathbb{K}}=\mathbb{Z}[\sqrt{-1}]$ is the ring of Gaussian integers, $d_{\mathbb{K}}=-4$, and $w_{\mathbb{K}}=4$. An integral Hermitian matrix $S \in \operatorname{Her}_{m}\left(\mathcal{O}_{\mathbb{K}}\right)$ is called even if $S[\mathbf{g}] \in 2 \mathbb{Z}$ for all $\mathbf{g} \in M_{m, 1}\left(\mathcal{O}_{\mathbb{K}}\right)$.

A main result of this note is as follows.

Theorem 3.1. Let $p$ be a prime number such that $p \equiv 3(\bmod 4)$, and let $S \in$ $\operatorname{Her}_{2}^{+}\left(\mathcal{O}_{\mathbb{K}}\right)$ be an even integral matrix such that $\operatorname{det}(S)=p$ (actually such a matrix $S$ exists for each prime $p$ satisfying $p \equiv 3(\bmod 4))$. If we define a sequence

$$
k_{m}=k_{m}(p):=2+(p-1) p^{m-1},
$$

then the corresponding sequence $G_{k_{m}}^{(n)}$ defines a p-adic Hermitian Eisenstein series. Moreover, we have

$$
\lim _{m \rightarrow \infty} G_{k_{m}}^{(n)}=\operatorname{genus} \Theta^{(n)}(S) .
$$

Namely, the p-adic Hermitian Eisenstein series $\lim _{m \rightarrow \infty} G_{k_{m}}^{(n)}$ becomes an ordinary Hermitian modular form of weight 2 and level $p$.

\section{Proof of the Main theorem}

We shall prove (3.1) by showing the coincidence of each Fourier coefficient of both sides. Let

$$
\lim _{m \rightarrow \infty} G_{k_{m}}^{(n)}=\sum \tilde{a}^{(n)}(H) q^{H}
$$

and

$$
\text { genus } \Theta^{(n)}(S)=\sum b^{(n)}(H) q^{H}
$$

be the Fourier expansions. Our goal is to prove the following identity:

$$
\tilde{a}^{(n)}(H)=b^{(n)}(H) .
$$

However, before proving this identity, we must investigate the coefficients $\tilde{a}^{(n)}(H)$ and $b^{(n)}(H)$. The coefficient $\tilde{a}^{(n)}(H)$ is the $p$-adic limit of $a_{k_{m}}^{(n)}(H)(m \rightarrow \infty)$. On 
the other hand, by the definition of the genus theta series, the coefficient $b^{(n)}(H)$ is given by

$$
\left(\sum_{i=1}^{h} \frac{A\left(S_{i}, 2 H\right)}{E\left(S_{i}\right)}\right) /\left(\sum_{i=1}^{h} \frac{1}{E\left(S_{i}\right)}\right),
$$

where

$$
A\left(S_{i}, 2 H\right):=\sharp\left\{X \in M_{2, n}\left(\mathcal{O}_{\mathbb{K}}\right) \mid{ }^{t} \bar{X} S_{i} X=2 H\right\} .
$$

First, we shall prove the following lemma.

Lemma 4.1. If $r:=\operatorname{rank}(H)>2$, then

$$
\tilde{a}^{(n)}(H)=b^{(n)}(H)=0 .
$$

Proof. Since $\operatorname{rank}(H)>\operatorname{rank}\left(S_{i}\right)=2$, the numbers $A\left(S_{i}, 2 H\right)$ vanish. This implies $b^{(n)}(H)=0$. Next, we prove that $\tilde{a}^{(n)}(H)=\lim _{m \rightarrow \infty} a_{k_{m}}(H)=0$. For this purpose, we set

$$
\mathbb{B}_{k}= \begin{cases}\frac{B_{k}}{k} & \text { if } k \text { is even, } \\ \frac{B_{k, \chi_{-4}}}{k} & \text { if } k \text { is odd, }\end{cases}
$$

where $B_{k}$ (resp. $\left.B_{k, \chi}\right)$ is the $k$-th Bernoulli number (resp. the $k$-th generalized Bernoulli number). If we pursue an argument similar to that of Boecherer [1] in the Hermitian modular case, then we see that the coefficient $a_{k_{m}}(H)$ can be written as

$$
a_{k_{m}}^{(n)}(H)=\prod_{i=0}^{r-1} \mathbb{B}_{k_{m}-i}^{-1} \cdot(\text { rational integer }) .
$$

(Refer also to [11, Proposition $4.7($ Case $S U)$.)

If $i \equiv 2(\bmod p-1)$, then

$$
\operatorname{ord}_{p}\left(\mathbb{B}_{k_{m}-i}^{-1}\right)=\operatorname{ord}_{p}\left(\frac{k_{m}-i}{B_{k_{m}-i}}\right) \geq 1
$$

(based on the classical theorem of von Staudt and Clausen). In particular, if $i=2$, then

$$
\operatorname{ord}_{p}\left(\mathbb{B}_{k_{m}-2}^{-1}\right)=\operatorname{ord}_{p}\left(\frac{p^{m-1}(p-1)}{B_{p^{m-1}(p-1)}}\right)=m .
$$

If $i \not \equiv 2(\bmod p-1)$, then there exists a $p$-adic number $d_{i} \in \mathbb{Q}_{p}$ satisfying

$$
\lim _{m \rightarrow \infty} \frac{k_{m}-i}{B_{k_{m}-i, \chi-4}}=d_{i}
$$

Therefore, we have

$$
\left.\operatorname{ord}_{p}\left(\mathbb{B}_{k_{m}-i}\right)=\operatorname{ord}_{p}\left(\frac{k_{m}-i}{B_{k_{m}-i, \chi_{-4}}}\right)=\operatorname{ord}_{p}\left(d_{i}\right) \quad \text { (for sufficiently large } m\right) .
$$

Combining these results, we have $\lim _{m \rightarrow \infty} \prod_{i=0}^{r-1} \mathbb{B}_{k_{m}-i}^{-1}=0$. This implies

$$
\lim _{m \rightarrow \infty} a_{k_{m}}^{(n)}(H)=0
$$

and completes the proof. 
From this result, for the purpose of proving (3.1), it suffices to prove that the identity (4.1) holds for the case $n=2$. Namely, our goal is reduced to the proof of the identity

$$
\tilde{a}^{(2)}(H)=b^{(2)}(H)
$$

for any $H \in \Lambda_{2}(\mathbb{K})$.

Lemma 4.2. If $\operatorname{rank}(H)=2$, then

$$
\tilde{a}^{(2)}(H)=b^{(2)}(H) .
$$

Proof. We shall first calculate $\tilde{a}^{(2)}(H)$. For this purpose, we introduce an explicit formula for $a_{k}^{(2)}(H)$ given by Krieg ([6], p. 678, Theorem and p. 679, Corollary):

$$
a_{k}^{(2)}(H)=\frac{4 k(k-1)}{B_{k} \cdot B_{k-1, \chi-4}} \sum_{0<d \mid \varepsilon(H)} d^{k-1} G_{\chi-4}\left(k-2, \frac{\operatorname{det}(2 H)}{d^{2}}\right),
$$

where

$$
\begin{aligned}
G_{\chi_{-4}}(s, N):= & \frac{1}{1+\left|\chi_{-4}(N)\right|}\left(\sigma_{s, \chi_{-4}}(N)-\sigma_{s, \chi_{-4}}^{*}(N)\right), \quad(s, N) \in \mathbb{Z}^{2}, \\
\sigma_{s, \chi_{-4}}(N):= & \sum_{0<d \mid N} \chi_{-4}(d) d^{s}, \quad \sigma_{s, \chi_{-4}}^{*}(N):=\sum_{0<d \mid N} \chi_{-4}(N / d) d^{s}, \\
& \varepsilon(H):=\max \left\{l \in \mathbb{N} \mid l^{-1} H \in \Lambda_{2}(\mathbb{K})\right\} .
\end{aligned}
$$

From this formula, we obtain

$$
\begin{aligned}
\tilde{a}^{(2)}(H) & =\frac{8}{B_{2, \chi_{-p}^{2}} \cdot B_{1, \chi_{-p}^{2} \chi_{-4}}} \sum_{\substack{0<d \mid \varepsilon(H) \\
(d, p)=1}} d \tilde{G}_{\chi_{-4}}\left(\frac{\operatorname{det}(2 H)}{d^{2}}\right) \\
& =\frac{48}{p-1} \sum_{\substack{0<d \mid \varepsilon(H) \\
(d, p)=1}} d \tilde{G}_{\chi_{-4}}\left(\frac{\operatorname{det}(2 H)}{d^{2}}\right),
\end{aligned}
$$

where

$$
\begin{aligned}
& \tilde{G}_{\chi_{-4}}(N):=\frac{1}{1+\left|\chi_{-4}(N)\right|}\left(\tilde{\sigma}_{\chi_{-4}}(N)-\tilde{\sigma}_{\chi_{-4}}^{*}(N)\right), \quad N \in \mathbb{Z}, \\
& \tilde{\sigma}_{\chi_{-4}}(N):=\sum_{\substack{0<d \mid N \\
(d, p)=1}} \chi_{-4}(d), \quad \tilde{\sigma}_{\chi_{-4}}^{*}(N):=\sum_{\substack{0<d \mid N \\
(d, p)=1}} \chi_{-4}(N / d) .
\end{aligned}
$$

Next, we shall calculate $b^{(2)}(H)$. By the Siegel formula in the case of Hermitian forms (cf. Braun [2, Otremba [9]), we have

$$
b^{(2)}(H)=\frac{1}{2} \prod_{q \leq \infty} \alpha_{q}(S, 2 H)
$$

where

$$
\begin{gathered}
\alpha_{q}(S, 2 H)=\lim _{a \rightarrow \infty} q^{-4 a} A_{q^{a}}(S, 2 H), \\
A_{q^{a}}(S, 2 H):=\sharp\left\{X \in M_{2}\left(\mathcal{O}_{\mathbb{K}}\right) \bmod q^{a} \mid{ }^{t} \bar{X} S X \equiv 2 H \bmod q^{a}\right\}
\end{gathered}
$$


and

$$
\alpha_{\infty}(S, 2 H)=\operatorname{det}(S)^{-2} \cdot \prod_{j=1}^{2} \frac{(2 \pi)^{j}}{{\sqrt{\left|d_{\mathbb{K}}\right|} \cdot(j-1) !}^{j}}=\frac{\pi^{3}}{p^{2}} .
$$

In order to calculate $\alpha_{q}(S, 2 H)$ more explicitly, we introduce the following notation: let $f(H)$ be the integer defined by $\operatorname{det}(2 H)=\varepsilon(H)^{2} \cdot f(H)$ and put

$$
\operatorname{det}(2 H)=\prod_{q: \text { prime }} q^{e_{q}}, \quad \varepsilon(H)=\prod_{q: \text { prime }} q^{\varepsilon_{q}}, \quad f(H)=\prod_{q: \text { prime }} q^{f_{q}} .
$$

We proceed the calculation of $\alpha_{q}(S, 2 H)$ by dividing the following three cases:

(i) $q$ is a prime number such that $q \neq 2, p$,

(ii) $q=p$,

(iii) $q=2$ (ramified prime).

For case (i), we have

$$
\alpha_{q}(S, 2 H)=\left(1-q^{-2}\right)\left(1-\chi_{-4}(q) q^{-1}\right) \sum_{l=0}^{\varepsilon_{q}} q^{l}\left(\sum_{m=0}^{e_{q}-2 l} \chi_{-4}(q)^{m}\right) .
$$

This follows from the main theorem in [7] (p. 235) (unramified case).

For case (ii), we obtain

$$
\alpha_{q}(S, 2 H)=\alpha_{p}(S, 2 H)= \begin{cases}\frac{(p+1)^{2}}{p} & \text { if } f_{p} \text { is odd }, \\ 0 & \text { otherwise. }\end{cases}
$$

This formula is derived from a formula by Hironaka [4 by setting $m=n=2$, $\lambda=\left(\varepsilon_{p}+f_{p}, \varepsilon_{p}\right)$, and $\mu=(1,0)$ in the main formula in Theorem 4 (4], p. 61).

For case (iii), we have

$$
\begin{aligned}
& \alpha_{2}(S, 2 H) \\
& =2^{2} \cdot\left(1-2^{-2}\right) \sum_{l=0}^{\varepsilon_{2}} 2^{l}\left\{1-\chi_{-4}\left(\operatorname{det}(2 H) / 2^{\varepsilon_{2}}\right)\left(1-\chi_{-4}\left(\operatorname{det}(2 H) / 2^{2 l}\right)^{2}\right)\right\} .
\end{aligned}
$$

As in case (i), this formula can be obtained from the main theorem in [7] (p. 235) (ramified case).

Now we assume that $\operatorname{ord}_{p}(\operatorname{det}(2 H))$ is odd, that is, the number $f_{p}$ is odd. In this case, by the above formulas, we have

$$
\begin{aligned}
b^{(2)}(H)= & \frac{1}{2} \cdot \prod_{q \leq \infty} \alpha_{q}(S, 2 H) \\
= & 2 \cdot \frac{1}{\zeta(2) L(1 ; \chi-4)} \cdot \frac{1}{\left(1-p^{-2}\right)\left(1-\chi_{-4}(p) p^{-1}\right)} \cdot \frac{(p+1)^{2}}{p} \cdot \frac{\pi^{3}}{p^{2}} \\
& \cdot \sum_{\substack{0<d \mid \varepsilon(H) \\
(d, p)=1}} d F\left(\frac{\operatorname{det}(2 H)}{d^{2}}\right) \\
= & \frac{48}{p-1} \sum_{\substack{0<d \mid \varepsilon(H) \\
(d, p)=1}} d F\left(\frac{\operatorname{det}(2 H)}{d^{2}}\right),
\end{aligned}
$$


where

$$
F(N):=\prod_{\substack{q: \text { prime } \\ q \neq p, 2}}\left(\sum_{m=0}^{\beta_{q}} \chi_{-4}(q)^{m}\right) \cdot \frac{1-\chi_{-4}\left(N^{\prime}\right)}{1+\left|\chi_{-4}(N)\right|}, \quad N=\prod q^{\beta_{q}}=2^{\beta_{2}} \cdot N^{\prime} .
$$

It is easy to see that $G_{\chi_{-4}}(s, N)$ has the following expression:

$$
G_{\chi_{-4}}(s, N)=\frac{1}{1+\left|\chi_{-4}(N)\right|} \prod_{q \neq 2}\left(\sum_{m=0}^{\beta_{q}} \chi_{-4}(q)^{m} q^{m s}\right)\left(1-\chi_{-4}\left(N^{\prime}\right) 2^{\beta_{2} s}\right) .
$$

Hence, we obtain

$$
F(N)=\tilde{G}_{\chi-4}(N)
$$

Therefore, if $\operatorname{ord}_{p}(\operatorname{det}(2 H))$ is odd, then $\tilde{a}^{(2)}(H)=b^{(2)}(H)$. On the other hand, if $\operatorname{ord}_{p}(\operatorname{det}(2 H))$ is even, we have $\tilde{a}^{(2)}(H)=b^{(2)}(H)=0$. This completes the proof of Lemma 4.2 .

Lemma 4.3. If $\operatorname{rank}(H)=1$, then

$$
\tilde{a}^{(2)}(H)=b^{(2)}(H)
$$

Proof. In the case where $\operatorname{rank}(H)=1$, we have

$$
a_{k}^{(2)}(H)=a_{k}^{(1)}(\varepsilon(H))=-\frac{2 k}{B_{k}} \sigma_{k-1}(\varepsilon(H)) .
$$

Thus, we obtain

$$
\tilde{a}^{(2)}(H)=-\frac{4}{B_{2, \chi_{-p}^{2}}} \sigma_{1, \chi_{-p}^{2}}(\varepsilon(H))=-\frac{4}{(1-p) B_{2}} \sum_{\substack{0<d \mid \varepsilon(H) \\(d, p)=1}} d=\frac{24}{p-1} \sum_{\substack{0<d \mid \varepsilon(H) \\(d, p)=1}} d .
$$

Next, we shall calculate $b^{(2)}(H)$ for the case in which $\operatorname{rank}(H)=1$. In this case, we have $2 H \sim\left(\begin{array}{cc}2 \varepsilon(H) & 0 \\ 0 & 0\end{array}\right)$ (unimodular equivalence). As in the case of $\operatorname{rank}(H)=2$, we can apply the Siegel formula and obtain

$$
b^{(2)}(H)=\prod_{q \leq \infty} \alpha_{q}(S, 2 \varepsilon(H)),
$$

where

$$
\begin{gathered}
\alpha_{q}(S, 2 \varepsilon(H))=\lim _{a \rightarrow \infty} q^{-3 a} A_{q^{a}}(S, 2 \varepsilon(H)), \\
A_{q^{a}}(S, 2 \varepsilon(H)):=\sharp\left\{X \in M_{2,1}\left(\mathcal{O}_{\mathbb{K}}\right) \bmod q^{a} \mid{ }^{t} \bar{X} S X \equiv 2 \varepsilon(H) \bmod q^{a}\right\}
\end{gathered}
$$

and

$$
\alpha_{\infty}(S, 2 \varepsilon(H))=\operatorname{det}(S)^{-1} \cdot 2 \varepsilon(H) \cdot \pi^{2}=\frac{2 \varepsilon(H) \pi^{2}}{p} .
$$

The value $\alpha_{q}(S, 2 \varepsilon(H))$ is given as follows.

If $q$ is a prime number satisfying $q \neq 2, p$ (which corresponds to case (i) in the proof of Lemma 4.2), then

$$
\alpha_{q}(S, 2 \varepsilon(H))=\left(1-q^{-2}\right) \sum_{l=0}^{\varepsilon_{q}} q^{-l}
$$


If $q=p$ (case (ii)), then

$$
\alpha_{p}(S, 2 \varepsilon(H))=\frac{1+p}{p^{1+\varepsilon_{p}}} .
$$

If $q=2$ (case (iii)), then

$$
\alpha_{2}(S, 2 \varepsilon(H))=2 \cdot\left(1-2^{-2}\right) \sum_{l=0}^{\varepsilon_{2}} 2^{-l} .
$$

Combining these formulas, we have

$$
\begin{aligned}
b^{(2)}(H) & =\prod_{q \leq \infty} \alpha_{q}(S, 2 \varepsilon(H)) \\
& =\frac{1}{\zeta(2) \cdot\left(1-p^{-2}\right)} \cdot \frac{1+p}{p^{1+\varepsilon_{p}}} \cdot 2 \prod_{q \neq p}\left(\sum_{l=0}^{\varepsilon_{q}} q^{-l}\right) \cdot \frac{2 \varepsilon(H) \pi^{2}}{p} \\
& =\frac{24}{p-1} \sum_{\substack{0<d \mid \varepsilon(H) \\
(d, p)=1}} d \\
& =\tilde{a}^{(2)}(H) .
\end{aligned}
$$

This completes the proof of Lemma 4.3.

Since $\tilde{a}^{(2)}\left(O_{2}\right)=b^{(2)}\left(O_{2}\right)=1$, we conclude the proof of the desired formula $(4.3)$.

\section{REFERENCES}

1. S. Boecherer, Über die Fourierkoeffizienten der Siegelschen Eisensteinreihen. Manuscripta Math. 45(1984), 273-288. MR0734842 (86b:11037)

2. H. Braun, Zur Theorie der hermiteschen Formen, Abh. Math. Sem. Univ. Hamburg 14(1941), 61-150. MR0004856 (3:70i)

3. H. Braun, Hermitian modular functions I, Ann. of Math. 50(1949), 827-855. MR0032699 $(11: 333 \mathrm{a})$

4. Y. Hironaka, Local zeta functions on Hermitian forms and its application to local densities, J. Number Theory 71(1998), 40-64. MR1631034(99e:11045)

5. H. Katsurada and S. Nagaoka, On some p-adic properties of Siegel-Eisenstein series, J. Number Theory 104(2004), 100-117. MR2021628(2004i:11042)

6. A. Krieg, The Maass spaces on the Hermitian half-space of degree 2, Math. Ann. 289(1991), 663-681. MR1103042 (93d:11051)

7. S. Nagaoka, An explicit formula for Siegel series, Abh. Math. Sem. Univ. Hamburg 59(1989), 235-262. MR1049898 (91i:11053)

8. S. Nagaoka, A remark on Serre's example of p-adic Eisenstein series, Math. Z. 235(2000), 227-250. MR:1795506 (2001m:11068)

9. G. Otremba, Zur Theorie der hermiteschen Formen in imaginär-quadratischen Zahlkörpern, J. Reine Angew. Math. 249(1971), 1-19. MR0318060 (47:6609)

10. J.-P. Serre, Formes modulaires et fonction zêta p-adiques, Lecture Notes in Math., Vol. 350, Springer, Berlin 1973, pp.191-268. MR0404145(53:7949a)

11. G. Shimura, On Eisenstein series, Duke Math. J. 50(1983), 417-476. MR0705034 (84k:10019)

Department of Mathematics, Kinki University, Higashi-Osaka, Osaka 577-8502, Japan

E-mail address: nagaoka@math.kindai.ac.jp 